\title{
Bilateral High-Origin Anterior Tibial Arteries and Its Clinical Importance
}

\author{
Sharma K, Haque MK, Mansur DI
}

Department of Anatomy

Kathmandu University School of Medical Sciences

Dhulikhel Hospital, Kathmandu University Hospital

Dhulikhel, Nepal.

Corresponding Author

Kalpana Sharma

Department of Anatomy

Kathmandu University School of Medical Sciences

Dhulikhel Hospital, Kathmandu University Hospital

Dhulikhel, Nepal

E-mail: sharmakalpu@yahoo.com

Citation

Sharma K, Haque MK, Mansur DI. Bilateral HighOrigin Anterior Tibial Arteries and Its Clinical Importance. Kathmandu Univ Med J 2012;37(1):88-90.

\begin{abstract}
Reported here is a case of bilateral high - origin anterior tibial arteries as detected during routine dissection of a 40 years old male cadaver at the Kathmandu University School of Medical Sciences, Kavre District, Nepal. The aim of the present study was to underline the clinical importance of high origin of anterior tibial artery from the popliteal artery.The high origin anterior tibial artery from the popliteal artery and its relations with the popliteus muscle is an important anatomical variation which should be paid attention during knee joint surgery, total knee arthroplasty and angiographic evaluations.
\end{abstract}

\section{KEY WORDS}

Anterior tibial artery, Popliteal artery, High origin

\section{INTRODUCTION}

The popliteal artery is the continuation of the femoral artery and crosses the popliteal fossa. It descends laterally from the opening in the adductor magnus muscle to the femoral intercondylar fossa, inclining obliquely to the distal border of the popliteus muscle, where it divides into the anterior and posterior tibial arteries. This division usually occurs at the proximal end of the asymmetrical crural interosseous space between the wide tibial metaphysis and the slender fibular metaphysis. ${ }^{1}$ The term high division of the popliteal artery is used when the first branch arises at or above the articular surface of the tibial plateau or if the bifurcation level is proximal to the lower border of the popliteus muscle. After the bifurcation, the anterior tibial artery usually runs dorsal to the popliteus muscle, passes between the heads of the tibialis posterior muscle and through the oval aperture in the proximal part of the interosseus membrane of the leg, on the anterior surface of the membrane to reach the extensor region of the leg, passing medial to the fibular neck and descends with the deep fibular nerve. Descending anteriorly on the membrane it approaches the tibia and, distally, lies anterior to it. At the ankle it is midway between the malleoli, and continues on the dorsum of the foot as the dorsalis pedis artery. ${ }^{1,2}$

\section{CASE REPORT}

During routine dissection of a 40 year old male cadaver (June 2011) at the Department of Anatomy, Kathmandu University School of Medical Sciences, a high - origin anterior tibial artery running along the ventral surface of popliteus muscle was observed on both sides. The high - origin anterior tibial artery descended between the popliteus muscle and the posterior surface of tibia after branching from the popliteal artery.The bifurcation level of 


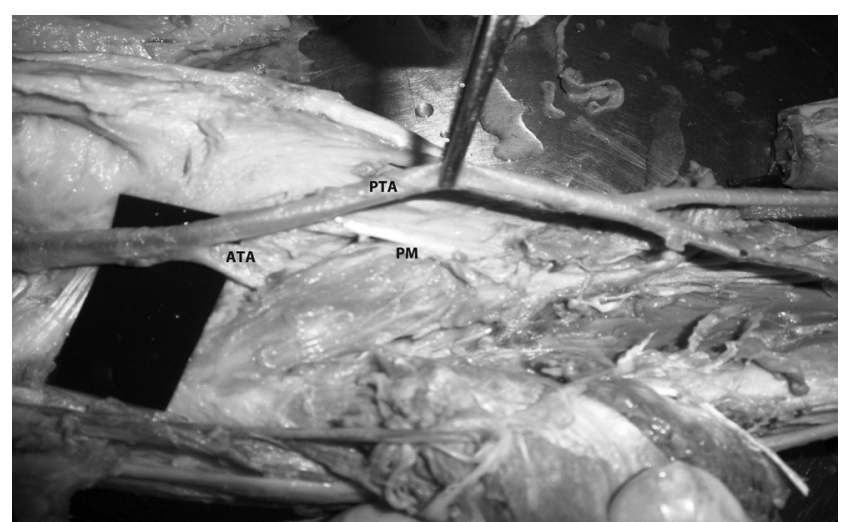

Figure.1 Dissection of Left popliteal region showing the highorigin left anterior tibial artery proximal to the upper border of popliteus muscle.

ATA: Anterior Tibial Artery

PTA: Posterior Tibial Artery

PM: Popliteus Muscle

the right popliteal artery into a high-origin anterior tibial artery and posterior tibial artery to the proximal border of popliteus muscle was $4.2 \mathrm{~cm}$. The bifurcation level of the left popliteal artery into a left high-origin anterior tibial artery and left posterior tibial artery to the proximal border of popliteus muscle was $2 \mathrm{~cm}$. The caliber of popliteal arteries, anterior tibial arteries and posterior tibial arteries were $8.0 \mathrm{~mm}, 2.9 \mathrm{~mm}$ and $4.0 \mathrm{~mm}$ respectively.

The high-origin anterior tibial artery descended between the popliteus muscle and posterior surface of the tibia after branching from the popliteal artery.

\section{DISCUSSION}

The popliteal artery may divide into its terminal branches proximal to the popliteus muscle, in which case the anterior tibial artery sometimes descends anterior to the muscle. There may be a true 'trifurcation' into anterior and posterior tibial and peroneal branches. Either the anterior tibial or the posterior tibial artery may be reduced or increased in size. The anterior tibial artery may be small but is rarely absent. Its function may be replaced by perforating branches from the posterior tibial artery or by the perforating branch of the peroneal artery. It occasionally deviates laterally, regaining its usual position at the ankle. It may also be larger than normal, in which case its territory of supply in the foot may be increased to include the plantar surface. ${ }^{1}$

Mauro MA, et al in 1988 studied 343 patients angiographically and observed a high-origin anterior tibial artery at the upper border of popliteus muscle and passing either superficially or deep to the popliteus muscle,2.3\% and a high-origin Anterior tibial artery passing either superficially or deep to the popliteus muscle with the peroneal artery arising from the anterior tibial artery, $0.9 \%{ }^{2}$

Kim D, et al assessed popliteal artery anatomy in 605 extrimities and the tibial arterial anatomy in 495

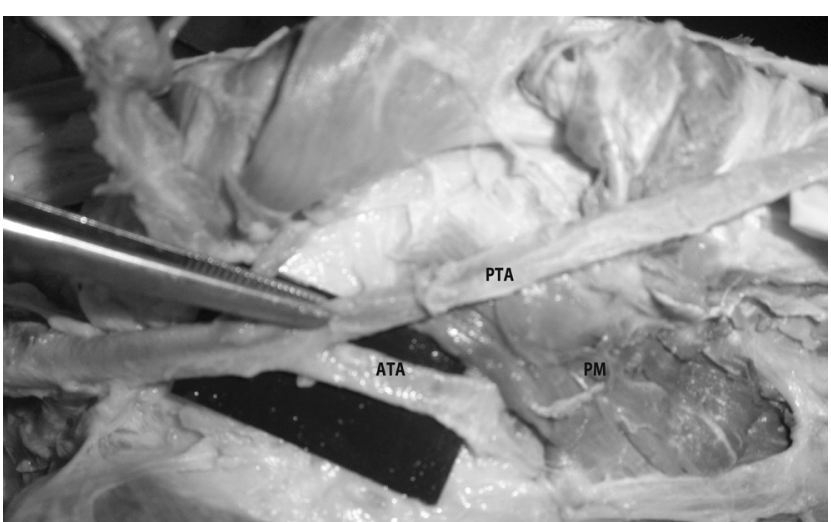

Figure.2 Dissection of right popliteal region showing the highorigin right anterior tibial artery proximal to the upper border of popliteus muscle.

ATA: Anterior Tibial Artery PTA:Posterior Tibial Artery PM: Popliteus Muscle

extrimities. Seventy five variant cases were identified. Normal branching of the popliteal artery was present in $92.2 \%$. Among the $7.8 \%$ incidence of varients, the majority $72 \%$ were either high-origin anterior tibial artery or a trifurcation pattern. ${ }^{3}$

Ertugrul $\mathrm{M}$, et al analyzed the popliteal branching patterns in 535 extremities (270 right, 265 left). The branching patterns were classified according to the level of branching and the presence of hypoplasia or aplasia of the distal branches.470 (88.1\%) limbs had a normal level of popliteal artery branching. High division of the popliteal artery was seen in 30 (5.6\%) limbs. 33 limbs (6.1\%) exhibited hypoplasia/aplasia of the distal branches. ${ }^{4}$

Szpinda M studied the origin, length and luminal diameter of anterior tibial artery in $\mathbf{4 6}$ men and 30 women by means of radiological and digital methods. The anterior tibial artery occurred most frequently $92.11 \%$ as a terminal branch of popliteal artery in its normal and high division. In the remainder $7.89 \%$, the anterior tibial artery arose from both the anterior tibioperoneal trunks. ${ }^{5}$

Tindall AJ, et al assessed the prevalence of proximal highorigin anterior tibial artery and its surgical significance. 100 knees were prospectively studied using colour Doppler ultrasonography. Prevalence of high-origin anterior tibial artery pattern was $6 \%{ }^{6}$

Sung-won K, et al analyzed the variations in branching of the popliteal artery by reviewing the femoral arteriograms. Digital subtraction angiographies of both the lower extremities were performed in 621 patients. Of the 1242 extremities, 1108 extremities (89.2\%) had normal branching pattern of the popliteal artery. The remaining 134 extremities (10.8\%) in 105 patients showed variant branching patterns of popliteal artery. ${ }^{7}$

Selda $Y$ reported a case of a high-origin anterior tibial artery running along the ventral surface of the popliteus muscle on the left side of 75 years old male cadaver. The bifurcation level of the popliteal artery into a high-origin anterior tibial 
artery and posterior tibial artery was $16.02 \mathrm{~mm}$ proximal to the upper border of the popliteus muscle. It was reported that high - origin anterior tibial artery branched at or above the level of articular surface of the tibial plateau at a ratio of $1.2 \%$ to $6 \% .^{8}$

The lower extremity arteries arise from two sources: sciatic artery and the femoral artery. Variability in the crural arteries depends on both the regression of the sciatic artery and also on the persistence of its junction with the primary femoral artery in the popliteal region. ${ }^{5}$ The popliteal artery arises as a sprouts from the axis artery at the proximal border of popliteus muscle, passes along the dorsal surface of the popliteus muscle and subdivides in the posterior tibial and primitive peroneal arteries which lie superficial to the tibialis posterior muscle.At the distal border of the popliteus muscle the axis artery provides a branch which passes ventrally above the interosseous membrane and is continued as the anterior tibial and dorsalis pedis artery.The embryonic vascular development determines the anatomic variability. Understanding the embryology and variant anatomy may have significant clinical implications. Vascular grafting and repair is indicated in a variety of disease processes, including atherosclerosis, emboli and trauma.
Anatomic variants will influence the surgical approach and dissection and the choice of suitable arterial graft sites. ${ }^{2}$ It is interesting for any anatomist to explore the possible embryological reasons behind the existence of anamolies. Anatomist have described that the branching patterns of the popliteal artery are related to the combinations of the sciatic artery and primary femoral arteries. ${ }^{9}$

\section{CONCLUSION}

An accurate anatomical knowledge of high-origin Anterior Tibial Artery from the popliteal artery and its relation to the popliteus muscle, is of immense help to the surgeons and orthopedicians for carrying out knee joint surgery, femoral distal end or proximal tibial/fibular fracture surgery, total knee arthroplasty and especially surgical interventions for popliteal artery aneurysms, which are the most common peripheral artery aneurysms due to atherosclerosis. This knowledge of anomalous origin of anterior tibial artery is also helpful for correct interpretation of angiographic reports involving such aberrant vessels.

\section{REFERENCES}

1. Standring S. Vascular supply and lymphatice drainage: Gray's Anatomy. 39th ed. New York: Elsevier Churchill livingstone; 2001. P. 1486.

2. Mauro MA, Jaques PF, Moore M. The popliteal artery and its branches: Embryological basis of normal and variant Anatomy, American Roentgen Ray Society. 1988; 150: 435-437.

3. Kim D, Orron DE, Skillman JJ. Surgical significance of popliteal arterial variants. A unified angiographic classification. Ann Surg 1989; 210:776-781

4. Ertugrul M, Halil D. Popliteal artery branching patterns detected by digital subtraction angiography. Turkish society of Radiology 2010; 17:80-83.

6. Tindall AJ, Shetty AA, James KD, Middleton A, Fernando KW. Prevalence and surgical significance of a high-origin ATA. J Orthop Surg 2006; 14:13-16.

7. Kil SW, Jung GS. Anatomical variations of the popliteal artery and its tibial branches. Cardiaovascular and interventional radiology. 32 (2); 233-240.

8. Yildiz S. A high-origin anterior tibial artery and its current clinical importance.international journal of anatomical variations 2010; 3:180-182.

9. Azian Abd,Das S.Anatomical insight into the normal and abnormal branching pattern of the popliteal artery.Surg Radiol Anat 31;563564.

5. Szpinda M. Digital-image analysis of the angiographic patterns of the popliteal artery in patients with aorto-iliac occlusive disease. Ann Anat 2006; 188:377-382. 